\title{
Thank You to Annals of Surgical Oncology Expert Reviewer Community
}

\author{
Mark Roh $^{1}$, Deborah Whippen ${ }^{2}$, and Charles Balch ${ }^{3}$ \\ ${ }^{1}$ UF Health Cancer Center Orlando, Orlando, FL; ${ }^{2}$ Annals of Surgical Oncology Editorial Office, North Fort Myers, FL; \\ ${ }^{3}$ University of Texas MD Anderson Cancer Center, Houston, TX
}

The Editors thank the experts listed below for serving as reviewers in 2017 for Annals of Surgical Oncology. The quality of the journal is sustained because of their efforts. We appreciate their critical and thorough assessments of submitted manuscripts, necessary for the substantial evaluation of papers. Their peer review service is part of the editorial assessment that provides benefit to both authors and readers for those papers that are accepted and published. The journal extends its gratitude with this acknowledgment.

Kim Aalders
Mark C. J. Aarts
Andrea Abbott
Daniel Abbott
Eddie Abdalla
Eddie Abdalla
Sherif Abdel-Misih
Firas Abdollah
Takashige Abe
Michael Abern
Ronan Abgral
John Abraham
Nadeem Abu-Rustum
René Adam
William P. Adams
Mehrad Adibi
Volkan Adsay
Prasad Adusumilli
Jayant Agarwal
Piyush Agarwal
Doreen Agnese
Syed Ahmad
Usman Ahmad
Hashim U. Ahmed

Kim Aalders

Mark C. J. Aarts

Andrea Abbott

Daniel Abbott

Eddie Abdalla

Eddie Abdalla

Sherif Abdel-Misih

Firas Abdollah

Takashige Abe

Michael Abern

Ronan Abgral

Nadeem Abu-Rustum

René Adam

Mehrad Adibi

Volkan Adsay

Prasad Adusumill

arwal

Piyush Agarwa

Syed Ahma

Hashim U. Ahmed

\author{
Khalil Ahmed \\ Gretchen Ahrendt \\ Nita Ahuja \\ Nicolas Ajkay \\ Javed Akhtar \\ Timothy Akhurst \\ Takashi Akiyoshi \\ Yasunori Akutsu \\ Adina Zeki Al Hazzouri \\ Waddah Al-Refaie \\ Hakan Alakus \\ Maarten Albersen \\ Kaled Alektiar \\ Mehrdad Alemozaffar \\ Giovanni Aletti \\ H. Richard Alexander \\ Zahraa AlHilli \\ Shadan Ali \\ Azra Alizad \\ Carmen Allegra \\ Martin S. Allen-Auerbach \\ Peter Allen \\ William Allum \\ Thomas Aloia
}

\author{
Nasser Altorki \\ Michael Alvarado \\ Petra Ambrosch \\ Mahul Amin \\ Eitan Amir \\ Moran Amit \\ Michele Ammendola \\ John Ammori \\ Benjamin Anderson \\ F. Andre \\ Eran R. Andrechek \\ Christoph Andree \\ Fernando Angarita \\ Christina Angeles \\ Roberto Angioli \\ Antonis C. Antoniou \\ Toru Aoyama \\ Patricia Aragon Han \\ Maria Arcila \\ Chandrakanth Are \\ Charlotte Ariyan \\ Jane Armer \\ Sarah T. Arron \\ Horacio Asbun
}

(C) Society of Surgical Oncology 2017

First Received: 31 October 2017;

Published Online: 22 November 2017

C. Balch

e-mail: balch@asoeditorial.org 
Caroline Aspord

Deanna Attai

Riccardo Audisio

Rebecca Auer

Rachel Aufforth

David August

Bruce Averbook

Eli Avisar

Itzhak Avital

Tomer Avraham

Umit Aydogmus

Hatem Azim

Daniel Azoulay

Jan P. Baak

Hideo Baba

Yoshifumi Baba

Kara Babaian

Marko Babjuk

Philippe Bachellier

Philippe Bachellier

Thomas Bachleitner-Hofmann

Floor Backes

Ketan K. Badani

Brian Badgwell

Sanjay Bagaria

Aditya Bagrodia

Glauco Baiocchi

Naoual Bakrin

Vinod Balachandran

Y. Balash

Glen Balch

Elizabeth Baldini

Marcos Ballester

Sa-Ik Bang

Sa-Ik Bang

Prof. Holger Bannasch

Andrew Barbour

Adam Barlow

Carlton Barnett

Daniel Barocas

Andrea Barrio

Parul Barry

David Bartlett

Ram Basak

Jose Baselga

Nesrin Tan Baser

Mark Basik

Claudio Bassi

Somprakas Basu

Pete Batra

Rich Battafarano

Da-Tian Bau

Sebastian Bauer
Todd Bauer

Joel Baumgartner

Marnelli A. Bautista-Quach

Nancy Baxter

Harry Bear

Georgia Beasley

Filip Bednar

Isabelle Bedrosian

Vanessa L. Beesley

Regina Beets-Tan

Geerard Beets

Kevin Behrns

Peter Beitsch

Jacques Belghiti

Yazid Belkacemi

S. Della Bella

Roser Belmonte

Merav Ben David

Ronny Ben-Avi

Kfir Ben-David

Shamgar Ben-Eliyahu

Edgar Ben-Josef

Sofiane Bendifallah

Nathalie Bendriss-Vermare

Toni Beninato

Julian Benito-Leon

Stephane Benoist

Karim Bensalah

Al Benson

David Bentrem

Brandon Bentz

Eren Berber

Wende Berg

Adam Berger

David Berger

Mitchel S. Berger

Ryan K. Berglund

Mariana Berho

Russell Berman

Mark Berry

Monica Bertagnolli

Sarah Besch

Michal J. Besser

Shanel Bhagwandin

Aditi Bhatt

Faiz Bhora

Stefan Bielack

Laura Biganzoli

Nicoletta Biglia

Lana Bijelic

Anton Bilchik

Karl Bilimoria

Thomas Birdas
Peyman Bjorklund

Dalliah Black

William Blackstock

Sarah Blair

Justiin Blasberg

Justin Blasberg

Jean Yves Blay

Jane Blazeby

Dan Blazer

Richard Bleicher

Joshua Bleier

Orin Bloch

Sharon Bober

Frederic Bodin

Ulrike Boehmer

Arne Boettcher

Daniel Boffa

Deborah A. Boggs

Sarah Bohndiek

Genevieve Boland

Richard Bold

Servet Bölükbas

Luigi Bonavina

Sylvie Bonvalot

Susan K. Boolbol

Brian Boone

Stephen A. Boorjian

Inne Borel Rinkes

Stefano Boriani

Ulrich Bork

Monique Bos

Dirk Bosch

Donald Bottaro

Judy Boughey

Michael Bouvet

Wilbur Bowne

Raghuram P. Boyapati

David Bracco

Carol Bradford

William Bradley

Mary Brady

Megan Branda

Savtaj Brar

Gennady Bratslavsky

Albert Breda

Rachel Brem

David Brenin

Meagan Brennan

Sandra Brennan

Noel T. Brewer

Noel T. Brewer

Abenaa Brewster

Emilio Bria 
Alejandro Bribriesco

Alberto Briganti

Robert Bristow

Vance Broach

Malcolm Brock

Stephen Broderick

Sabine Brookman-May

Gabriel Brooks

Gina Brown

Lisa Brown

Adam Brufsky

Paul Brunault

C. Buchbender

Elizabeth Buckley

Alessandro Buda

Markus Buechler

Ralph Bueno

William Burfeind

Maximilian Burger

Jocelyn Burke

Bryan Burt

Ronald W. Busuttil

Charles Butler

Jean Butte

David Byrd

James Byrd

Federico Cacciapuoti

Luca Calabrese

Dario Callegaro

Glenda Callender

Ellen Calogeras

Katherina Calvillo

Diego Calvisi

Ernest Camp

Chris Campbell

H. E. Campbell

Ian G. Campbell

Alfredo Campenni

Emel Canbay

David J. Cangemi

Martin Canis

Geoffroy Canlorbe

Donald M. Cannon

Jennifer Cannon

Daniel J. Canter

Robert Canter

Umberto Capitanio

Martyn Caplin

L. Capussotti

Stefano Caramuta

Fabio Carboni

Kenneth Cardona

Francesco Carli
Tobias Carling

Grant Carlson

Denise Carneiro-Pla

Leah Y. Carreon

Philip Carrott

Philip Carrott

W. Carter

Sally Carty

Jose Enrique Casal Nunez

Paolo Casali

Pedro Cascales Campos

Peter Cashin

Stephen Cassivi

Stephanie Caterson

Abigail Caudle

José Antonio Cavalhero

Wim Ceelen

Andrea Cercek

Bulent Cetin

Charles Cha

Anees Chagpar

Kaisorn Chaichana

Sricharan Chalikonda

Mael Chalret du Rieu

Ronald Chamberlain

Carlos Chan

Jimmy Yu Wai Chan

Kevin Chan

Manish Chand

Zhang Chang-hua

Andrew Chang

Daniel Chang

David Chang

David W. Chang

David Z. Chang

Edward Chang

George Chang

Hsien-Kun Chang

Joe Chang

Sam Chang

Steven Chang

Celia Chao

Yin-Kai Chao

William Chapman

Anil Chaturvedi

Ian Chau

H. Chave

Mariana Chavez-MacGregor

Akhil Chawla

Jacques E. Chelly

Allen Chen

Allen M. Chen

Amy Y. Chen
Chiung-Nien Chen

Guoan Chen

Haiquan Chen

Helen Chen

Herbert Chen

Junqiang Chen

Keneng Chen

Ling Chen

Ming-Yuan Chen

Ronald C. Chen

Rufu Chen

Steven Chen

Tianxiang Chen

Wei-liang Chen

Yen-Lin Chen

Yuanjia Chen

Edward Cheng

Ming-Huei Cheng

Shih-Ping Cheng

Zandra Cheng

Daniel Cherqui

Tan To Cheung

Andrea Cheville

Dennis Chi

Vito Chiantera

Akira Chikamoto

Laura Chin-Lenn

Bill Chiu

Chien-Hung Chiu

Philip Chiu

T. W. Chiu

Clifford Cho

Kwan Ho Cho

Dong Wook Choi

Eun-Young (Karen) Choi

Joon Young Choi

Mihye Choi

Moon Seok Choi

Sang-Yong Choi

Michael Choti

Haroon Choudry

Louis Chow

Oliver Chow

Henrik Kidmose Christensen

Kathleen Christians

Carrie Chu

Danny Chu

Quyen Chu

Terence Chua

Linus Chuang

S. Jeff Chueh

Yoon Chun

Yun Shin Chun 
Alice Chung

Christine Chung

Dai Chung

Man Ki Chung

Woong Youn Chung

James M. Church

Fabio Cianchi

David Cibula

Maciej Ciebiada

Emanuele Cigna

Thomas Clancy

Jonathan Clark

Bryan Clary

J. M. Classe

Pierre Clavien

Pierre-Alain Clavien

Sean Cleary

Mark Clemens

Krishna Clough

Jordan Cloyd

Natalie Coburn

Alistair Cochran

Mark Codner

Hiram Cody

Debbie Cohen

Marc Cohen

Sarah L. Cohen

Daniel Coit

Matthew Colman

Christos Colovos

Amy Colwell

Kevin Conlon

Kevin Conloon

Saxon Connor

Claudius Conrad

Giovanni Conzo

David Cooke

Jonathan Cools-Lartigue

Amanda Cooper

Matthew Cooperberg

Suzanne Coopey

Edward Copeland

Kimberly Corbin

Niall M. Corcoran

Erin Cordeiro

Peter Cordeiro

M. Cordes

Janice Cormier

Lorraine Cornwell

Carlos Corvera

Nicholas G. Cost

Meagan Costedio

Gregory Cote
Michael Cowher

John Cox

Jennifer Cracchioli

Aimee Crago

Chris Crane

Margaret Crivello

John Cuaron

Alessandro Cucchetti

Steven Curley

James Cusack

Amy Cyr

Michael D'Angelica

Jonathan D'Cunha

Xavier Benoit D'Journo

Domenico D'Ugo

Fadi Dahdaleh

Benedict Daly

John Daly

MacKenzie Daly

Samantha Damude

Chengxue Dang

Gail Darling

Elizabeth David

Michael Davies

John Davis

Noelle Davis

Kristopher M. Day

Remco de Bree

Robbert de Haas

Ignace de Hingh

Ignace $\mathrm{H}$. de Hingh

Vincent de Meijer

Jorma J. de Ronde

Eduardo de Santibañes

Amy Degnim

Marco Del Chiaro

Marcela Del Rio

Thomas Delaney

Leigh Delbridge

Leigh Delbridge

Frank J. DellaCroce

Keith Delman

Ronald DeMatteo

Michael Demeure

Jason Denbo

Jeremiah Deneve

Carsten Denkert

Chadrick Denlinger

Marcello Deraco

Maryam Derogar

Frank Detterbeck

Matthias Dettmer

E. Gene Deune
Eric J. Devor

Mojgan Devouassoux

Elisabeth Dexter

Elisabeth Dexter

Mark Dickson

Paxton Dickson

Marie-Danièle Diebold

Emilia Diego

David Dietz

Jill Dietz

Juan J. Diez

Mary Dillhoff

Sean Dineen

Frederick Dirbas

Marius Distler

Elijah Dixon

J. Michael Dixon

Gerard Doherty

Ryuichiro Doi

James Dolan

James Donahue

Timothy Donahue

XinQi Dong

William Dooley

Tanya B. Dorff

Lesly Dossett

Majella Doyle

Anthony E. Dragun

Francois Dravet

Jeffrey Drebin

Jeffrey Drebin

Florence Duffaud

Quan-Yang Duh

Quan-Yang Duh

Frédéric Dumont

Geoffrey Dunn

Christy Dunst

Uma Duvvuri

Paula J. Duxbury

Timothy Eberlein

Michael Ebright

James Economou

Stephen Edge

David Edholm

Barish Edil

Melanie Edwards

Bernhard Egger

Michael Egger

Behfar Ehdaie

Matthias Eiber

Fritz Eilber

Daniel B. Eisen

Graeme Eisenhofer 
B. Ejlertsen

Mahmoud El-Tamer

Dominique Elias

Joshua Ellenhorn

Joshua Ellenhorn

Beatrix Elsberger

Mohamed A. Elshaikh

Yassir Eltahir

Jean Emond

Shigenobu Emoto

Itaru Endo

Cathy Eng

Oliver Eng

Jonathan I. Epstein

Lillian Erdahl

Ane Gerda Eriksson

Anna Gerda Eriksson

Mert Erkan

Derek Erstad

Martin Espinosa-Bravo

Jesus Esquivel

Laura Esserman

David Euhus

Young Gyu Eun

Maristella Evangelista

Andrew J. Evans

D. Gareth Evans

Douglas Evans

Samer Ezziddin

Matthew Facktor

Thomas Fahey

Bridget Fahy

Mark Fairweather

Massimo Falconi

Massimo Falconi

Songqing Fan

Jugao Fang

Qi-Gen Fang

Wen-Tao Fang

Grant T. Fankhauser

William Faquin

Olivier Farges

Mark Faries

Farhood Farjah

Jeffrey Farma

Omar Farouk

D. Farwell

Martin Fassnacht

Barry Feig

Seth Felder

Sheldon Feldman

Volker Fendrich

Carlos Fernandez-del Castillo
Felix Fernandez

Hiran Fernando

Alessandro Ferrero

Robert Ferris

Cristina Ferrone

Cristina R. Ferrone

Juan J. Fibla

Ryan Fields

Ryan Fields

Martin Filipits

Samuel Finlayson

Marco Fiore

John Fischer

Fergal Fleming

Nicole Fleming

James Fleshman

Erik Folch

Yuman Fong

Samuel Ford

Meghan Forster

Jason Foster

Christina Fotopoulou

Keith Fournier

Valerie Francescutti

Todd Francone

Todd Francone

Timothy Frankel

Rachel Freedman

Richard Freeman

Phoebe Freer

Christian Freudlsperger

Michael Frumovitz

Bryan Fuchs

Kazumasa Fujitani

Yoshiyuki Fujiwara

David Fuks

Elena Fumagalli

Susanne Füssel

Michele Gadd

Wolfgang Gaertner

David K. Gaffney

Henning Gaissert

Georgios Gakis

T. Gamblin

Tong-Joo Gan

Giorgio Gandaglia

Kristen Ganjoo

Ian Ganly

Yuzhen Gao

Giuseppe Garcea

Antonio Garcia Fernandez

Julio Garcia-Aguilar

Julio Garcia-Aguilar
Evan Garfein

Patrick Garvey

Jennifer Gass

Andrew A. Gassman

Wolfgang Gatzemeier

Guillaume Gauchotte

Paul Gauger

Somali Gavane

Haim Gavriel

Paola Gazzaniga

E. Geijsen

Hans Gelderblom

David Geller

Mary Gemignani

Jeanine Genkinger

Oreste Gentilini

Jean-Pierre Gerard

Tamer Ghanem

Abdel-Hamid A. Ghazal

Michelle Ghert

Francois Ghiringhelli

Ziv Gil

Anthony Gill

Erin Gillaspie

Luca Giovanella

Armando Giuliano

Pier Giulianotti

Olivier Glehen

Ana Gleisner

Bengt Glimelius

Gabriel Glockzin

Robert Glynne-Jones

Vincent Gnanapragasam

Jennifer Gnerlich

Tony Godfrey

Lucas Goense

Diane Goéré

Matthew Goetz

Thorsten Oliver Goetze

Ofer N. Gofrit

Brian Goh

Ziya Gokaslan

Jason Gold

Marc Gollub

Mehra Golshan

Daniel Gomez

Paolo Gontero

Santiago Gonzalez Moreno

Ricardo Gonzalez

Jin Mo Goo

Karyn Goodman

Martin Goodman

Jennifer Gordetsky 
Emre Gorgun

L. Gottwald

Anand Govindarajan

Sharad Goyal

Ian Grady

Paul Graham

Frederic Grannis

Clive Grant

Gwenaelle Gravis

Richard Gray

Adele Green

Vicky Green

Caprice Greenberg

Alexander Greenstein

Rachel Greenup

Michael Griffin

Valerie Grignol

Gregory Grillone

Rob Grimer

Christoph Grimm

Jan Grimm

Lars Grimm

Andrea Grin

Stephen Grobmyer

Eric Grogan

Raymon Grogan

Alessandro Gronchi

Caroline Gronnier

Shawn Groth

Axel Grothey

Travis Grotz

Amelia Grover

Elizabeth Grubbs

Viktor Grünwald

Michael Grynberg

B. Ashleigh Guadagnolo

Hamza Guend

Marlon Guerrero

Jose Guillem

Andrew Gumbs

Niraj Gusani

Vadim Gushchin

Uwe Güth

Graeme Guthrie

David Gyorki

Patrick Ha

Jennifer S. Haas

Rick Haas

Elizabeth Habermann

Daniel Habermehl

Thilo Hackert

Christina Hackl

Tufia Haddad
Stephan Haerle

Bruce Haffty

Jeroen Hagendoorn

Karl E. Haglund

Christiane Hakim

Ashleigh Halderman

Carolyn Hall

Peter S. Hall

Susan Hallbeck

Julie Hallet

Geoffrey G. Hallock

Moustapha Hamdi

Trevor Hamilton

Chet Hammill

Dale Han

Guohong Han

Ho-Seong Han

Wonshik Han

Matthew Hanasono

Andrew Hanby

George Hanna

Nader Hanna

Stéphane Hans

Noboru Hara

Tomohiko Hara

Avital Harari

Joseph T. Hardwicke

Iraj Harirchi

Jay Harris

Lawrence Harrison

Andreas Hartkopf

Dana Hartl

C. E. A. Hartmann

Kiyoshi Hasegawa

Masaharu Hata

Ioannis Hatzaras

Eric Hau

Alexander R. Haug

Bruce Haughey

William Hawkins

William Hawkins

Andrea Hayes-Jordan

Alex Haynes

Jin $\mathrm{He}$

Mark Healy

Jason Hearns

Elham Hedayati

A. Heidenreich

C. William Helm

Alan Hemming

Michael Henderson

Helen Heneghan

Bryan Hennessy
Jonathan Hensel

Martee Hensley

Fernando Herbella

Joseph Herman

Roberto Hernandez-Alejandro

Enrique Hernandez

Harry Herr

Virginia Herrmann

Daniel Herzig

Martin Heslin

Akira Hida

Tina Hieken

Michael L. Hinni

Alice $\mathrm{Ho}$

Chuong Hoang

Steven Hochwald

Richard Hodin

Harald Hoekstra

Sarah Hoffe

John Hoffman

Jürgen Hoffmann

Johannes Hofland

Leo Hofland

Michael Hofman

Wayne Hofstetter

Peter Hohenberger

Emma Holliday

Chris Holsinger

Ginger Holt

Goro Honda

Kohei Honda

Joon Pio Hong

Theodore Hong

Charles Honoré

Andrew Hoover

Raymund Horch

Akira Horii

Hisakazu Hoshi

Michael House

Nehmat Houssami

Nehmat Houssami

Gilles Houvenaeghel

Marissa Howard-McNatt

J. Howard

John Howard

Thomas Howard

James Howe

Carrie Hruska

Michael Hsiao

Cary Hsu

Meier Hsu

James $\mathrm{Hu}$

Chang-Ming Huang 
James Huang

Steven Huang

Tao Huang

Kay Huebner

David Hughes

David T. Hughes

Kevin Hughes

Marybeth Hughes

Steven Hughes

Maarten Hulshof

Olivier Humbert

Richard Hummel

Scott Hundahl

Brian Hung-Hin Lang

Jung-Jyh Hung

Kelly Hunt

Steve Hunt

Arti Hurria

Georg Hutterer

Jutta Huvila

E. Shelley Hwang

Jin-Hyeok Hwang

John Hyngstrom

Woo Jin Hyung

Calogero Iacono

Roberto Iacovelli

David Iannitti

Alexia Iasonos

Daisuke Ichikawa

Kamran Idrees

Tetsuo Ikeda

Brian Ilfeld

Masayuki Imamura

Massimo Imazio

Issei Imoto

Teruo Inamoto

Daniel J. Indelicato

Yusuke Inoue

Y. Irani

Jonathan Irish

Abid Irshad

James Isbell

Junichiro Ishioka

Mitsuru Ishizuka

Akihiro Ito

Hiroaki Ito

Hiromichi Ito

Tetsuhide Ito

Yasuhiro Ito

Srinivas Ivatury

N. Gopalakrishna Iyer

Jakob Izbicki

Kouji Izumi
Namiki Izumi

Ruth H. Jack

Lisa Jacobs

Isabelle Jaffre

Ajay Jain

Michael Jaklitsch

James Jakub

Ted James

Georg Jancke

Shareef Jandali

David Jaques

William Jarnagin

Dawn Jaroszewski

Nicolás Jarufe

Nora Jaskowiak

Karl Jauch

Mahsa Javid

Sara Javid

Milind Javle

Shiva Jayaraman

Davi Jayne

Jacqueline Jeruss

J. Milburn Jessup

Kim Jessup

Mike Jewett

Sanghoon Jheon

Long Jiao

Qiu Jinsong

Blair Jobe

Blair Anderson Jobe

Jason Johanning

Nathalie Johnson

Fabian Johnston

Jon Jonasson

Thomas Julian

Young Ho Jung

Theodor Junginger

Kerstin Junker

Matias Jurado

Hiroaki Kajiyama

Yoshihiro Kakeji

Kennichi Kakudo

Matthew Kalady

Kapila Kalakota

Shingo Kanaji

Mitsuro Kanda

Tatsuo Kanda

Emad Kandil

John Kane III

Gyeong Hoon Kang

Sung-Bum Kang

Yoon-Koo Kang

Chia-Hung Kao
Huang-Kai Kao

Georgios Karagkounis

Giorgos Karakousis

Paul Karanicolas

Manoj Kumar Karmakar

Nolan Karp

Martin Karpeh

Ahmed Kaseb

Bernd Kasper

Chikatoshi Katada

Hitoshi Katai

E. Katerinaki

Hiroyuki Kato

Michael Kattan

Matthew Katz

Steven Katz

Howard Kaufman

Koji Kawaguchi

Mark L. Kayton

Electron Kebebew

Bastian Keck

Gary Keeney

Gisela Keller

Mark Kelley

Kaitlyn Kelly

Nancy Kemeny

Michael Kendrick

Gregory Kennedy

Timothy Kennedy

Tara Kent

Kenneth Kesler

Susan Kesmodel

Hermann Kessler

Marco Kesting

Seema Khan

Onkar Khullar

Adam S. Kibel

Masahiro Kikuchi

Shane Killeen

Brigid Killelea

John Kiluk

Anthony Kim

Byung Sik Kim

Dae Yong Kim

Dong Kwan Kim

Gwang Ha Kim

Heung Yeol Kim

Ho-joong Kim

Hong Kim

Hong Jin Kim

Hyung-Ho Kim

Jin Kim

Jin You Kim 
John Kim

Joseph Kim

Joseph Kim

Jun-Gi Kim

Kyubo Kim

Lawrence Kim

Min Kim

Min Chan Kim

Nam-Kyu Kim

S. Samuel Kim

Samuel Kim

Seon-Hahn Kim

Seong-Jang Kim

Seungwon Kim

Simon Kim

Song Kim

Sun Wook Kim

Sun-Whe Kim

Sung-Hoon Kim

Tae Hee Kim

Tae Hyuk Kim

Tae-You Kim

Wun-Jae Kim

Yong-Hee Kim

Yoon Jun Kim

Young-Hoon Kim

Cindy Kin

Tammy Kindel

Jonathan King

Judy W. King

Tari King

T. Peter Kingham

Tetsushi Kinugasa

Ravi P. Kiran

David Kirsch

Yuko Kitagawa

Hiroshi Kitamura

Andreas Kjær

Yvonne L. B. Klaver

Jorg Kleeff

Fredrik Klevebro

V. Suzanne Klimberg

Joachim Klode

Michael Kluger

Jeffrey Kneisl

Hisataka Kobayashi

Tadao K. Kobayashi

Yasuhiro Kodera

Viktor H. Koelzer

Fumitaka Koga

Yoon Woo Koh

Takuya Koie

Norihiro Kokudo
Shuhei Komatsu

Amanda Kong

Ingmar Königsrainer

Tsuyoshi Konishi

Tsuyoshi Konishi

Bon Seok Koo

David Kooby

Bas Koolen

Katherine Kopkash

Michael Koretz

Lale Kostakoglu

Lei Kou

Luiz Kowalski

David Kozono

Jeffrey Krane

Seth Krantz

Dennis Kraus

Lutz Kretschmer

Mark G. Kris

Laura Kruper

Ja Hyeon $\mathrm{Ku}$

Naoshi Kubo

Takeshi Kubota

Henry Kuerer

Moshim Kukar

Swati Kulkarni

Rakesh Kumar

Jolanta Kunikowska

Chikara Kunisaki

Yur-Ren Kuo

Michael Kupferman

Shigeki Kusamura

Ryoji Kushima

Boris Kuvshinoff

Hiroyuki Kuwano

Mary Kwaan

Jin Young Kwak

David Kwon

King Kwong

Daniel Labow

Ollivier Laccourreye

Marc Ladanyi

Jennifer LaFemina

Jesper Lagergren

Jesper Lagergren

Michael Lagios

Guy Lahat

Eric C. H. Lai

Lily Lai

Stephen Lai

Terry Lairmore

Vincent W. T. Lam

Laura Lambert
Donald L. Lamm

Jeffrey Landercasper

Ron Landmann

Luca Landoni

Jerome Landry

Brian Lang

Julie Lang

Rupert Langer

Cord Langner

Michael Lanuti

Cathryn Lapedis

Michael Large

Christine Laronga

David Larson

Gail A. Laughlin

Christophe Laurent

Simon Law

Alexander Lazar

Huong Le-Petross

Emmanuèle Lechapt-Zalcman

Fabrice Lecuru

Rebecca Leddy

Ann Lee

Anne W. M. Lee

Anne W. M. Lee

Byrne Lee

Byung-Joo Lee

Clara Lee

Hyuk-Joon Lee

Hyun Moo Lee

Jae-Lyun Lee

James Lee

Jay Lee

Jeffrey Lee

Jeffrey Lee

Jeonghun Lee

King-Teh Lee

Kyung Soo Lee

Linda Lee

M. Catherine Lee

Nancy Lee

M. W. H. Leenders

Mario Leitao

Marilyn Leitch

Valerie Lemaine

Javier Lendoire

Eric Lentsch

Eric Lentsch

Roberto Leon Ferre

Maria Leonardi

Stanley Leong

Trevor Leong

Jeffrey Leow 
Jan Lerut

Edward Levine

John Lew

James S. Lewis

Valarae Lewis

Chien-Feng Li

$\mathrm{Fu}-\mathrm{Yu} \mathrm{Li}$

Guojun Li

Guoxin Li

Jia Li

Shasha Li

Yan Li

Zhiyu Li

Zibo Li

Ching-Chung Liang

Han Liang

Lianming Liao

Stanley Liauw

Moishe Liberman

Sender Liberman

Steven Libutti

Stuart Lichtman

Gerrit-Jan Liefers

Joon Seok Lim

Jung-won Lim

Yun Sung Lim

Jen-Der Lin

Jules Lin

Michael Lin

Sam Lin

Steven Lin

Brenessa LIndeman

Joerg Lindenmann

David Linehan

John Lipham

David Liska

Virginia Litle

Jennifer Litton

Eric Liu

Fei-Fei Liu

Ji-Yan Liu

Lu-Ming Liu

Ping Liu

Marc Lobbes

John A. LoGiudice

Chris Lominska

Michael T. Longaker

Alessandra Longhi

Richard Longnecker

Ana López-de-Andrés

Caroline Lopez

Laura Lorenzon

Pei-Jen Lou
Wen-Hui Lou

Donald Low

Donald Low

Michael Lowe

Andrew Lowy

Nir Lubezky

Carrie Lubitz

Anthony Lucci

James Luketich

Lars Lundell

Cynthia Ma

Huiyan Ma

Monique Maas

Teresa Macarulla

Khaled M. Madbouly

David Madoff

Robert Madoff

Ugwuji Maduekwe

Yoshihiko Maehara

Haider Mahdi

Najjia Mahmoud

Armeen Mahvash

Hai-Qiang Mai

Shishir Maithel

Anirban Maitra

Grace Mak

Ray Mak

Marty Makary

Ajay Maker

Robert Maki

Vicky Makker

Eleftherios Makris

George Malietzis

Mary Malucci

Joshua Mammen

Harvey Mamon

Terry Mamounas

Gianpiero Manca

Divneet Mandair

G. S. Mannu

Paul Mansfield

John Mansour

Jose Manuel Baena-Canada

Jodi Maranchie

Karen Marcus

Julie Margenthaler

Christophe Mariette

Patricia Marino

Michael Marinovich

Sheraz Markar

Arnold Markowitz

John Marks

Frederik Marmé
Daniele Marrelli

Jennifer Marti

Matthew Martin

Robert Martin

Steve Martinez

Steve R. Martinez

Dipen Maru

Tadahiko Masaki

Holly Mason

Nader Massarweh

Amit Mathur

Evan Matros

Satoru Matsuda

Soeren Mattke

Matthias May

Shuhei Mayanagi

Horacio F. Mayer

Justin Maykel

Skye Mayo

Haggi Mazeh

Judith McCart

Martin McCarter

Colleen McCarthy

W. Glenn McCluggage

Yarrow McConnell

Denise Elizabeth McCormack

Kelly McCoy

Lachlan McDowell

David McFadden

Sean McGuire

Kandace McGuire

Kandace P. McGuire

Chris McHenry

Sarah McLaughlin

Kelly McMasters

Donald McMillan

Joshua Meeks

Ari Meguerditchian

Robert Meguid

Reza Mehran

A. M. Mehta

Lieuwe Melchers

Marcovalerio Melis

Laleh Melstrom

Robin Mendelsohn

William Mendenhall

Jane Mendez

Christine Menetrier-Caux

Nipun Merchant

Funda Meric-Bernstam

Ryan Merkow

Ryan P. Merkow

Neal Meropol 


\begin{tabular}{|c|c|c|}
\hline Robert Merritt & Masaru Morita & Kalyana Nandipati \\
\hline Jennifer E. Mersereau & Arden Morris & Amol Narang \\
\hline Liljana Mervic & David Morris & Kailash Narayan \\
\hline Craig Messick & Monica Morrow & Govindarajan Narayanan \\
\hline Ingrid Meszoely & Dion Morton & Steven Narod \\
\hline Kelly Metcalfe & Paul Mosca & Garrett Nash \\
\hline Rajendra B. Metgudmath & Anne Mosenthal & Atsushi Nashimoto \\
\hline Peter Metrakos & Fuyuhiko Motoi & Katie Nason \\
\hline Tim Meyer & Kazuyoshi Motomura & Hari Nathan \\
\hline Michael Meyers & Samdeep Mouli & Shoji Natsugoe \\
\hline James Mezhir & Vladimir Mouraviev & Neema Navai \\
\hline Michael Michael & Thomas Mücke & Christopher Neal \\
\hline Markku Miettinen & Jennifer Mueller & Luca Negosanti \\
\hline Mira Milas & Christina Muijs & Jonas A. Nelson \\
\hline Barbra Miller & Amol Mujoomdar & Jonathan Nesbitt \\
\hline Kimberly Miller & Robert Mulholland & David Neskey \\
\hline Jeffrey Milsom & John Mullen & Carolyn Nessim \\
\hline Koshi Mimori & John Mullinax & Alfred Neugut \\
\hline Tommaso Mineo & Sean Mulvihill & Susan Neuhaus \\
\hline Thomas Miner & Goo-Hyun Mun & Susan Neuhausen \\
\hline Andrea Minervini & Alexandre Munhoz & Heather Neuman \\
\hline Bruce Minsky & Pamela N. Munster & Pippa Newell \\
\hline Roberto N. Miranda & Shuji Murakami & Elliot Newman \\
\hline Joseph Misdraji & Yoshiaki Murakami & Lisa Newman \\
\hline Subhasis Misra & Tomohiro Murakawa & Martin I. Newman \\
\hline Brad Mitchell & Rajmohan Murali & Joanne Ngeow \\
\hline Timur Mitin & Thibaut Murez & Dao Nguyen \\
\hline Elizabeth Mittendorf & Adam Murphy & Ninh Nguyen \\
\hline Akira Miyajima & Declan Murphy & Toan Nguyen \\
\hline Akira Miyajima & Sudish Murthy & Frank Nichols \\
\hline Hideaki Miyake & Peter Muscarella & Markus Niemeyer \\
\hline Hiroshi Miyamoto & Matthew Mutch & Hitoshi Niikura \\
\hline Isao Miyashiro & Robert Mutter & Yuri Nikiforov \\
\hline Hiroshi Miyata & Fabio Muttin & Magnus Nilsson \\
\hline Shugo Mizuno & Terence Myckatyn & Naris Nilubol \\
\hline Jacob Moalem & Ioannis Mylonas & Motoki Ninomiya \\
\hline Harveshp Mogal & Nima Nabavizadeh & Yutaka Nishiwaki \\
\hline Daniela Molena & Hidenari Nagai & Aviram Nissan \\
\hline Jeffrey Moley & Hiroaki Nagano & Mirko Nitsche \\
\hline Daniel J. Mollura & Hiroaki Nagano & Masakuni Noguchi \\
\hline Arash Momeni & Masato Nagino & Yuki Nohara \\
\hline Adili Moming & David Nagorney & Hiroaki Nomori \\
\hline Shalini Moningi & Maurice Nahabedian & Bernard Nordlinger \\
\hline Tracy-Ann Moo & Zoher M. Naja & Jeffrey Norton \\
\hline Summer D. Moon & Eric Nakakura & Stephanie Nougaret \\
\hline Eric Moore & Masafumi Nakamura & Philipp Nuhn \\
\hline Brendan Moran & Yasuni Nakanuma & Steven Nurkin \\
\hline Meena Moran & Yuichiro Nakashima & J. Nyhof-Young \\
\hline Daniel M. Moreira & Meijin Nakayama & Roisin O'Cearbhaill \\
\hline Todd M. Morgan & Attila Nakeeb & Elizabeth O’Flynn \\
\hline Masaki Mori & Faina Nakhlis & Brian O’Sullivan \\
\hline Ryo Morimura & Kenjiro Namikawa & Ciara O'Sullivan \\
\hline Masaru Morita & Govind Nandakumar & Inge-Marie Obdeijn \\
\hline
\end{tabular}




\section{Kjell Oberg}

Vincent Obias

Do-Youn Oh

Ryuji Ohashi

Takashi Ohtsuka

Hiroshi Okabe

Kutluk Oktay

Karl Oldhafer

Jeam Haroldo Oliveira Barbosa

David Ollila

John A. Olson

Go Omura

Mark Onaitis

Gary Onik

Shinji Ono

Celine Op den Kamp

Susan Orloff

Steffen Ormanns

Mark Orringer

Tomo Osako

Raymond Osarogiagbon

Fahima Osman

Iman Osman

Aytekin Oto

Eigo Otsuji

Katja Ott

Laura Ottini

Fang-Shu Ou

Pascal Owusu-Agyemang

Toshifumi Ozaki

Soji Ozawa

Tsuyoshi Ozawa

Enver Ozer

Murat Ozturk

Karel Pacak

Andrew Page

Natin Pagedar

Reetesh Pai

Yong Han Paik

Daniel Palmes

Jia-Min B. Pang

Alessandro Paniccia

Yves Panis

Mary Panjari

Sam Pappas

Sareh Parangi

Rajiv Parikh

Bernard Park

James Park

Seong-Heum Park

S. J. Parker

Ian F. Parney

Ann Partridge
Janice Pasieka

Sandro Pasquali

Guillaume Passot

J.-J. Patard

Dhaval Patel

Ketan Patel

Ketan Patel

Snehal Patel

Sunil Patel

Urjeet A. Patel

Urjeet A. Patel

Sujata Patil

Caitlin Patten

Philip Paty

Anna Pavlick

Timothy Pawlik

I. Benjamin Paz

Prema Peethambaram

Arjun Pennathur

George Peoples

Manuel Pera

Daniel Perez

Rodrigo Perez

Christos Perisanidis

Nancy Perrier

Niek Peters

Walter Peters

Ivy Petersen

Carrie Peterson

Iva Petkovska

Fausto Petrelli

Firas Petros

Henrik Petrowsky

Curtis Pettaway

Christian Peyre

Giao Phan

John Phay

Philip Philip

Joseph Phillips

Arnoldo Piccardo

Martin Pichler

Allan Pickens

Jean-Yves Pierga

Phillip Pierorazio

Guillaume Piessen

Alessio Pigazzi

Melissa Pilewskie

Venu Pillarisetty

Jose Pimiento

Peter Pinto

Pompiliu Piso

Pompiliu Piso

Henry Pitt
Susan Pitt

Guillaume Ploussard

John Plukker

Barbara Pockaj

Patricio Polanco

Tom Polascik

Blaze Polite

Raphael Pollock

David Polsky

Stephan Polterauer

Rodney Pommier

Irinel Popescu

Hendrik Van Poppel

Matthew Porembka

John Porterfield

Mitchell Posner

Emily Postma

George Poultsides

Stephen Povoski

Simon Powell

Kumar Prabhash

C. S. Pramesh

Veronique Preat

Jarrod Predina

Jason Prescott

Katharine A. R. Price

H. Miles Prince

Richard Prinz

Giuseppe Procopio

Ajay Puri

Varun Puri

Lajos Pusztai

Joe Putnam

Motaz Qadan

Jian Qiu

Michael Quentin

Denis Querleu

Veronique Quillien

Christiano Quintini

Philip Quirke

Harry Quon

N. A. Quraishi

Jennifer Racz

Stefano Radaelli

Laszlo G. Radvanyi

Salvatore Raffa

Marco Raffaelli

C. Raffaitin

Nuh Rahbari

Nuh Rahbari

Ganesh Raj

E. A. Rakha

Pedro Ramirez 
Majeed Rana

Jason R. Randall

R. Lor Randall

Ganesh Rao

Roshni Rao

Stephen M. Rao

Edoardo Raposio

Asif Rashid

Mamoon Rashid

L. Rasmussen

Gaiane Rauch

Rebecca Read

Tom Read

Kevin Reavis

Timothy R. Rebbeck

Rishindra Reddy

Sanjay Reddy

Sushanth Reddy

Nicholas P. Reder

John Rees

Meredith Regan

Scott Regenbogen

William Regine

Douglas Reintgen

Christoph Reissfelder

Ottavio Rena

Michele Reni

Matthew Resnick

Sha'Shonda Revels

Marc Reymond

Marsha Reyngold

John Reynolds

John V. Reynolds

Scott Reznik

Deborah Rhodes

Taylor Riall

Michelle Riba

Reitan Ribeiro

Dario Ribero

Dario Ribero

Rocco Ricciardi

David Rice

Colin Richards

Jeremy Richmon

Jens Ricke

Michael Rink

Brian Rinker

Robert Ripley

Nabil Rizk

Monica Rizzo

Geoffrey Robb

K. Robbins

D. J. Robinson
James Rocco

Alexandra Roch

Flavio Rocha

Sjoerd Rodenhuis

David Roder

Miguel A. Rodriguez-Bigas

Michael Roehrl

Kevin Roggin

Jong-Lyel Roh

Mark Roh

Christina Roland

Benjamin Roman

Sanziana Roman

Cristina L. Ronchi

Jan Roodenburg

Matti Rookus

Frederik Roos

Bart Rose

Charles Rosen

Laura Rosenberger

Robert Roses

Gary Ross

Merrick Ross

Emma Rossi

Gedge Rosson

Philippe Rouanet

Mathieu Rouanne

Margaret Roubaud

Morgan Roupret

Pascal Rousset

Koen Rovers

Campbell Roxburgh

Richard Royal

Warren Rozen

Laura Rubbia-Brandt

Mark A. Rubin

Isabel Rubio

Jens Rückert

Kathryn Ruddy

Eric Rullier

I. B. Runnebaum

Maria Russell

Emiel Rutgers

Piotr Rutkowski

Iris Rutten

David Ryan

Min-Hee Ryu

Antonio Sa Cunha

Anne M. Saaristo

Michael Sabel

Ramin Sadeghi

Hiroshi Saeki

Sait Sager
Yendamuri Sai

Susumu Saigusa

Michel Saint-Cyr

Michel Saint-Cyr

Bruno Sainz

M. S. Sajid

Yoshihiro Sakamoto

Yasuhiko Sakata

Takashi Sakatani

Evis Sala

Ritu Salani

Ramón Salazar

Riad Salem

Ronald Salem

Derya Kivrak Salim

Rubens Sallum

Peter Salmon

Jarmo Salo

George Salti

Maurizio Salvati

Paolo Sammartino

Tarik Sammour

Marta Sánchez-Carbayo

Marta Sánchez-Carbayo

Takeshi Sano

Markku Santala

Fabio Santanelli

Alfredo Santillan

Armando Sardi

Chitra Sarkar

Inderpal (Netu) Sarkaria

Juan Sarmiento

Michael Sarr

Shigeru Sasaki

Mitsuru Sasako

Sohei Satoi

Brian Saunders

Alain Sauvanet

Silviu Sbiera

Hani Sbitany

Courtney Scaife

Marco Scarpa

Olivier Scatton

Erik Schadde

Tracey Schefter

Douglas Scherr

A. Schiettecatte

Leif Schiffmann

Paul Schipper

Todd Schlachter

Francisco Schlottmann

Heinz Schmidberger

Carl Schmidt 
Jan Schmitges

Caroline Schmitt

Bryan Schneider

David Schneider

Sebastian Schoppmann

Deborah Schrag

David Schreiber

David Schrump

Mathew Schuchert

Richard Schulick

Joseph Schwab

Myron Schwartz

Theresa Schwartz

Roderich Schwarz

Daniel Sciubba

Charles Scoggins

Christopher Scott

Christopher Seder

T. Seiwert

Jesse Selber

Luke Selby

David Seligson

Stephen Sener

Sashendra Senthi

Maheswari Senthil

Boris Sepesi

Yasuyuki Seto

Alessandro Sgambato

Miraj Shah-Khan

Jay Shah

Manish Shah

Shimul Shah

Armin Shahrokni

Simona Shaitelman

Robert Shamberger

Basel Sharaf

Shahrokh Shariat

Jyotirmay Sharma

Piyush Sharma

Ranjna Sharma

Elizabeth Shaughnessy

Christiana Shaw

Kyle Sheetz

Feng Shen

Jeannie Shen

Jianxiong Shen

Perry Shen

Robert Shen

Wen Shen

Vanessa B. Sheppard

David Sher

Chanjuan Shi

Qian Shi
Jinru Shia

Hiroaki Shiba

David Shibata

Masatsune Shibutani

Alexander Shifrin

Tomoyuki Shimabukuro

Hideaki Shimada

Kazuaki Shimada

Yoshihisa Shimada

Tomoharu Shimizu

Yasuhiro Shimizu

Tadakazu Shimoda

Jae Il Shin

Junichi Shindoh

Nobuo Shinohara

Akihiro Shiotani

Mikio Shiozawa

Ken Shirabe

Yoshio Shirai

Katsutoshi Shoda

Joseph Shrager

Ravi Shridhar

Shailesh Shrikhande

Craig Shriver

Lawrence Shulman

Jason Sicklick

Rebecca Siegel

Robert J. Siegel

Carlie Sigel

Smita Sihag

Diane Simeone

Geert Simkens

Matthew Simmons

Rache Simmons

Giuseppe Simone

David Sindram

Samuel Singer

S. Singh-Carlson

Allan Siperstein

Rebecca Sippel

Eila Skinner

Joseph Skitzki

Geoffrey Sklar

Ted Skolarus

Roman Skoracki

Craig Slingluff

M. A. Smach

Barbara Smith

Fraser Smith

J. Smith

James Smith

Myles Smith

Petur Snaebjornsson
Sam Snyder

Jimmy So

Kevin Soares

Carmen Solarzano

Claudio Sole

Pamela Soliman

Michael Solomon

Carmen Solorzano

Mark Soloway

Antonio Sommariva

Josh Sommer

Vernon Sondak

Cheryn Song

Kyo Young Song

Christopher Sonnenday

Toyooki Sonoda

Atilla Soran

Atilla Soran

Halfdan Sørbye

Francesco Soria

Julie Sosa

Amine Souadka

Cigdem Soydal

Angela Spanu

Scott Spear

Michelle Specht

Elena Sperk

Aldona Spiegel

John Spiliotis

Andrew Spillane

Silvia Stacchiotti

Zsofia Stadler

Steven Stain

Charles Staley

Daniela Stan

Milan Stankovic

John Stauffer

Scott Steele

Jennifer Steiman

Jennifer A. Stein

Gary Steinberg

Jennifer Steve

John Stewart

Robert Sticca

Brendon Stiles

Karyn Stitzenberg

Luca Stocchi

Oliver Stoeltzing

Alexander Stojadinovic

Michael Stotz

Nicole Stout

Steaven Strasberg

Dirk C. Strauss 
Cathy Streutker

Luc Strobbe

Vivian Strong

Jonathan Strosberg

Christopher Strzalka

Cord Sturgeon

Hiroo Suami

Preeti Subhedar

Paul Sugarbaker

Sonia Sugg

Teiichi Sugiura

Jules Sumkin

Hai-bo Sun

Jiaming Sun

Steve Sun

Zhe Sun

R. Sundaresan

Janice Sung

Nilda Suslu

Jeff Sussman

Pasithorn Suwanabol

Kenji Suzuki

Yasuyuki Suzuki

Robert Svatek

Sandra Swain

Carol Swallow

Scott Swanson

Raeshell Sweeting

Elizabeth Swisher

Mark Sywak

Janio Szklaruk

Josep Tabernero

Kyung Tae

Lorraine Tafra

Melissa Taggart

Alphonse Taghian

Shyh-Kuan Tai

Emanuela Taioli

Toshio Takagi

Daisuke Takahari

Hidenori Takahashi

Shinichiro Takahashi

Kyoichi Takaori

Tadatoshi Takayama

Hiroyuki Takei

Akinobu Taketomi

Hiroya Takeuchi

Yoshifumi Takeyama

Shuji Takiguchi

Mia Talmor

Yasuhiro Tamaki

Hung-Jui (Ray) Tan

Kay See Tan
Kenneth Tanabe

Shinji Tanaka

Jun Tang

Cullen Taniguchi

Pieter J. Tanis

Bachir Taouli

William Tap

Christoph Tausch

Hin Ngan Tay

Julia Tchou

Lisette Te Velde

Paris Tekkis

Larissa Temple

Walley Temple

Britt ten Wolde

Patty L. Tenofsky

Melissa Teo

Melissa Teo

Alicia Terando

Masanori Terashima

Mediget Teshome

Stamatios Theocharis

A. Thomas

Charles Thomas

Jeremy Thomas

Pascal Thomas

Ryan Thomas

Alastair Thompson

Geoffrey Thompson

John Thompson

Margaret Thompson

Carrie Thoms

Rodolphe Thuret

Peter Thuss-Patience

I. Tinhofer

Yasushi Toh

Yuji Toiyama

Alper Toker

Masanori Tokunaga

Matthew Tollefson

James Tomlinson

Betty Tong

King-Hung Tong

Harrys Antonio Torres

Keila Torres

Mylin Torres

Guido Torzilli

Phuoc Tran

T. Treasure

Giorgio Treglia

Jose Trevino

Pauline Truong

Mark Truty
Susan Tsai

Wen-Sy Tsai

Raymond Tsang

Jennifer Tseng

Ling-Ming Tseng

William Tseng

Alexander Tsivian

Daisuke Tsugawa

Koji Tsuta

Kiran Turaga

Kiran K. Turaga

Olivier Turrini

Benjamin Tuschy

Michael Tuttle

Todd Tuttle

Tove Tvedskov

Douglas Tyler

Ching-Wei Tzeng

Robert Udelsman

Mamoru Uemura

Alexis Ulrich

Dmytro Unukovych

Celine M. Vachon

Alexander Vahrmeijer

Jayant S. Vaidya

Efsevia Vakiani

Renato A. Valdés Olmos

Stephanie Valente

Michele Valmasoni

Alexander van Akkooi

George Van Buren

Frits van Coevorden

Cornelis van de Velde

Willemien van de Water

Margriet Van der Heiden-van der Loo

Theodorus van der Kwast

Pieter van der Sluis

Nicole van Grieken

Thomas M. van Gulik

Winan van Houdt

Raquel van la Parra

Hanneke van Laarhoven

Fijs van Leeuwen

Krijn P. van Lienden

Peter van Rossum

Stijn van Weert

Bob van Wely

Kimberly Van Zee

Ara Vaporciyan

Gauri Varadhachary

Thomas Varghese $\mathrm{Jr}$

Aislinn Vaughan

Jean-Nicolas Vauthey 
Hidde Veenstra

Nirmal Veeramachaneni

Vic Velanovich

Annelies Vellekoop

Alan Venook

Cornelis Verhoef

Vic Verwaal

John Vetto

John Vetto

Michael Vezeridis

Eric Vibert

Selwyn Vickers

Marco De Vincentiis

Giuseppe Visconti

Brendan Visser

Alessandro Vitale

Christiane Voit

Charles Vollmer

Margaret von Mehren

Gunter von Minckwitz

Lena von Schuckmann

Michael von Wolff

Konstantinos Votanopoulos

Andrea Wahner Hendrickson

Go Wakabayashi

Evan Walgama

Markus Wallwiener

Matthew Walsh

Bing-Yen Wang

Dian Wang

Hailun Wang

Hsiu-Po Wang

Huamin Wang

Jian Wang

Jiping Wang

Sam Wang

Tracy Wang

Yanong Wang

Yi Zarn Wang

Yong-Sheng Wang

Zhen-ning Wang

Zhenning Wang

Irene Wapnir

Jennifer Wargo

Kenneth Washburn

Nabil Wasif

Jun Watanabe

Kazuhiro Watanabe

Masayuki Watanabe

Shunichi Watanabe

Toshiaki Watanabe

Jeffrey Wayne

Sharon Weber
Walter Weder

Jon Wee

Alice Wei

Benjamin Wei

Iris Wei

Katie E. Weichman

Ronald Weigel

Allison Weisbrod

Martin Weiser

Matthew Weiss

Jürgen Weitz

Christopher S. Wendel

Kai Wermker

A. Gabriella Wernicke

Brian West

Lynn M. Westphal

Steven Wexner

Michael Weyant

Young E. Whang

Rebekah White

Richard White

Dennis Wicherts

Pawel Wiechno

Daniel Wiener

Dennis Wigle

Bas Wijnhoven

Traci A. Wilgus

Scott Wilhelm

Lee Wilke

Michelle Wilkinson

Neal Wilkinson

Breelyn Wilky

Shawna Willey

Stephen Williams

Matthew Wilson

David J. Winchester

Joshua Winer

Eric Winquist

Emily Winslow

Desmond Winter

Jordan Winter

Juan Wisnivesky

Christopher Wolfgang

Jan Wong

Sandra Wong

Yanghee Woo

Christopher Wood

John C. Wood

Francis P. Worden

Stephanie Worrell

Curtis Wray

Jason Wright

Chen-Tu Wu
Jie Wu

Shaoyuan $\mathrm{Wu}$

Xiang-Yuan Wu

L. Wudel

Jay Wunder

Jiaqing Xiang

Bang-Wen Xie

Xian-Jie Xie

Rui-hua Xu

Evanguelos Xylinas

Hiroshi Yabusaki

Rona Yaeger

Diane Yamada

Yasuhide Yamada

Rin Yamaguchi

Junji Yamamoto

Shigeru Yamamoto

Shinya Yamamoto

Tomohisa Yamamoto

Tomoki Yamano

Hiroki Yamaue

Katsuhiko Yanaga

Masahiro Yanagawa

Cheng-Chien Yang

Han-Kwang Yang

Stephen Yang

Tsung-Lin Yang

Wei Yang

Yue Yang

Zhaohai Yang

Rhonda Yantiss

James Yao

Katharine Yao

Bharat Yarlagadda

Catheryn Yashar

Takushi Yasuda

David Yates

Ding Ye

Ding-wei Ye

Michael Yeh

Tina Yen

Charles Yeo

Heather Yeo

Chin A. Yi

Connie Yip

Linwah Yip

Akira Yokomizo

Yukata Yonemura

Harry Yoon

Sam Yoon

Akihiko Yoshida

Kazuhiro Yoshida

Takaki Yoshikawa 
Keiji Yoshinaga

Y. Nancy You

He Yu-long

Chia-Cheng Yu

Evan Y. Yu

Ke-Da Yu

Peirong $\mathrm{Yu}$

Peiwu YU

Xiao-Min Yu

Haluk Yuzugullu

Emmanouil Zacharakis

Jonathan Zager

Hisamitsu Zaha

Victor Zannis
Victor Zaydfudim

Herbert Zeh

Martha Zeiger

Michael Zelefsky

Amelia Zelnak

Yoh Zen

Nicole Zern

J. Y. Zhang

Jie Zhang

Li Zhang

Tao Zhang

Ronghua Zhao

Dong Bing Zhao

Haitao Zhao
Ming Zhao

Wenrui Zhao

Lei Zheng

Li Zhou

Qin Zhou

Zhiwei Zhou

Angela J. Ziebarth

Richard Zigeuner

Israel Zighelboim

Israel Zighelboim

Camilla Zimmermann

Amer Zureikat

Nicholas Zyromski 